\title{
A NOVEL MICROSTRIP DUAL-BAND BANDPASS FILTER USING DUAL-MODE SQUARE PATCH RESONATORS
}

\section{Kenneth S. K. Yeo* and Augustine O. Nwajana}

School of Architecture, Computer and Engineering, University of East London, UK

\begin{abstract}
Dual-mode square patch resonator is well known in the design of a single band quasi-elliptic bandpass filter response. Here, the dual-mode square patch resonator is employed to achieve a dualband bandpass filter. A 6 pole dual-band bandpass filter response with 3 poles at each passband will be presented. The dual-band filter also exhibits a transmission zero between the two passbands. A detailed discussion on the design procedure together with the simulation and experimental results will be presented.
\end{abstract}

\section{INTRODUCTION}

In recent years, there has been growing interest in the design of passive microwave filters. This interest is more profound when it comes to the design of multi-band filters. Dual-band bandpass filter design is increasingly important with the recent rapid growth, development, and progress in multi-band wireless communication systems. Dualband bandpass filters are also useful for isolating a small section of a frequency spectrum within a wider frequency spectrum.

Planar technology, i.e., the microstrip technology, has played, and is still playing a major role in the field of microwave circuits and systems. Planar dual-band microstrip bandpass filters have been widely research and are widely reported in the literatures [1-5].

In this paper, a novel planar dual-band bandpass filter using microstrip dual-mode square patch resonators will be presented. The microstrip dual-mode square patch resonator has been widely researched [6-9] for designing a single band bandpass filter with quasielliptical response for high selectivity applications. Employing dualmode patch resonators in filter design is an excellent way to miniaturize

\footnotetext{
Received 3 December 2012, Accepted 19 January 2013, Scheduled 23 January 2013

* Corresponding author: Kenneth Siok Kiam Yeo (k.yeo@uel.ac.uk).
} 
the filter layout. The dual-mode resonator filters are normally half the size of a similar single mode resonator filter. This is mainly due to the fact that each dual-mode resonator can provide two resonating poles in the filter response. Hence, the number of resonators required for an $n$-number of pole filter is $n / 2$, where $n$ is a positive even number integer. Filters that employed dual-mode resonators in their design will result in compact filter configuration, i.e., small in size.

In this paper, the design concept of achieving a dual-band bandpass filter using dual-mode square patch resonators will be presented. A detailed design procedure starting from a normalized lowpass filter circuit model to a microstrip dual-band dual-mode square patch bandpass filter design layout will be demonstrated by employing successive frequency transformations and circuit conversions. The dual-band filter presented will have the following specification:

- Center frequency, $f_{o}: 4185 \mathrm{MHz}$.

- Center frequency of lower passband, $f_{o, B P L}: 4021 \mathrm{MHz}$.

- Center frequency of upper passband, $f_{o, B P U}: 4356 \mathrm{MHz}$.

- Passband return loss: $20 \mathrm{~dB}$.

- Fractional Bandwidth of lower passband, $\mathrm{BW}_{L}: 4 \%$.

- Fractional Bandwidth of upper passband, $\mathrm{BW}_{U}: 4 \%$.

The dual-band filter will be achieved using 6 resonators. Therefore, the filter is a 6 th order filter, i.e., 6 poles with 3 poles located on each passband. The filter also exhibits a transmission zero between the two passbands. This filter can be used for isolating a frequency spectrum between $4100 \mathrm{MHz}$ to $4270 \mathrm{MHz}$, which falls within a wider communication spectrum of $3922 \mathrm{MHz}$ to $4460 \mathrm{MHz}$.

\section{CIRCUIT MODEL}

The circuit model of a dual-band filter can be established from a normalized Chebyshev low-pass filter, which is shown in Figure 1. The normalized low-pass parameters can be determined from [10,11]. To simplify the transformation process, the normalized ladder lowpass filter can be transformed into a normalized low-pass filter which consists of shunt reactive components only by employing admittance inverters as shown in Figure 2. The admittance inverter ( $J$-inverter) of the transformed low-pass filter can be determined using Equation (1).

$$
\begin{aligned}
J_{01}^{\prime} & =J_{n, n+1}^{\prime}=1 \quad \text { where } n=\text { order of the filter } \\
J_{m, m+1}^{\prime} & =\sqrt{\frac{g_{1}^{2}}{g_{m} g_{m+1}}} \quad \text { where } m=1,2, \ldots n
\end{aligned}
$$




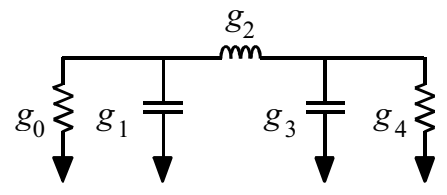

Figure 1. A standard normalized ladder form low-pass filter, reproduced courtesy of The Electromagnetics Academy

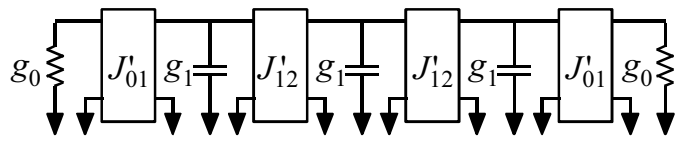

Figure 2. A normalized low-pass filter with shunt reactive components only, reproduced courtesy of The Electromagnetics Academy

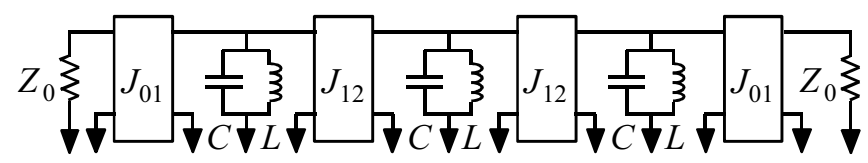

Figure 3. A Chebyshev filter circuit model with identical shunt of $L C$ resonators with $J$-inverters, reproduced courtesy of The Electromagnetics Academy

To establish a circuit model for a dual-band bandpass filter, the normalized low-pass filter is further transformed into a bandpass filter, which consists of shunt resonator only, i.e., shunt of inductor $(L)$ and capacitor $(C)$ to ground. In order to achieve a dual-band circuit model, the bandpass filter is designed to have the same passband ripple as the dual-band filter with a bandwidth which is equal to the combined bandwidth of the two passbands [5]. This can be achieved easily by scaling each of the shunt capacitors into shunt resonators using the standard low-pass to bandpass transformation [10,11]. Since all the shunt capacitors in Figure 2 are equal to $g_{1}$, the bandpass filter transformation is only involved in transforming a single parameter, i.e., $g_{1}$, to a shunt of $L C$ resonator. To scale the impedance of the model from the normalized impedance, i.e., $g_{0}$, to $Z_{o}$, all the $J$-inverters have to be divided by $Z_{o}$ as given in (2). The bandpass filter circuit model with identical shunt resonators is shown in Figure 3.

$$
J_{m, m+1}=\frac{J_{m, m+1}^{\prime}}{Z_{o}} \quad \text { where } m=0,1,2, \ldots n
$$

Once the bandpass filter model is established, a dual-band bandpass filter can be achieved by coupling each of the shunt resonators with an additional identical shunt resonator through a $J$-inverter, $J_{11^{\prime}}$, 
as shown in Figure 4 . The $J$-inverter, $J_{11^{\prime}}$, can be determined from the center frequencies of the two desired passbands, $f_{o, B P L}$ and $f_{o, B P U}$, by using (3).

$$
J_{11^{\prime}}=k_{11^{\prime}} \sqrt{\frac{C}{L}} \quad \text { where } k_{11^{\prime}}=\frac{f_{o, B P U}^{2}-f_{o, B P L}^{2}}{f_{o, B P U}^{2}+f_{o, B P L}^{2}}
$$

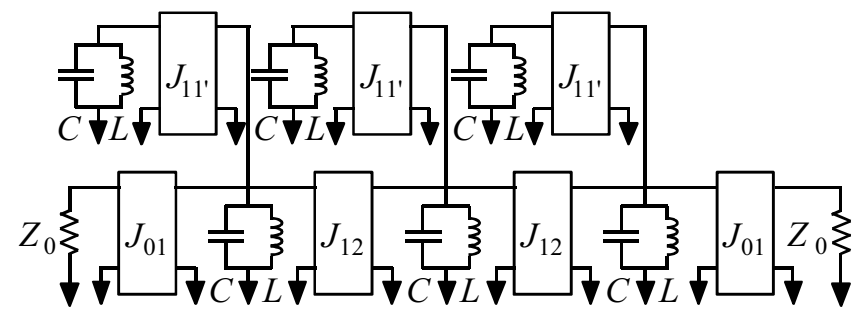

(a)

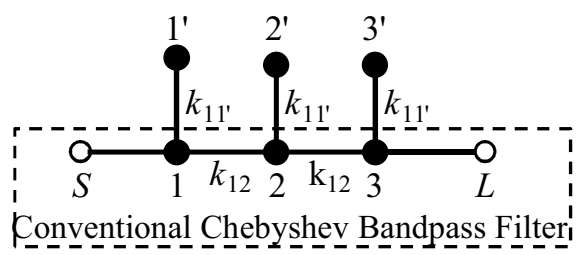

(b)

Figure 4. (a) Circuit model of a dual-band bandpass filter with all identical $L C$ resonators. (b) The equivalent node diagram, reproduced courtesy of The Electromagnetics Academy

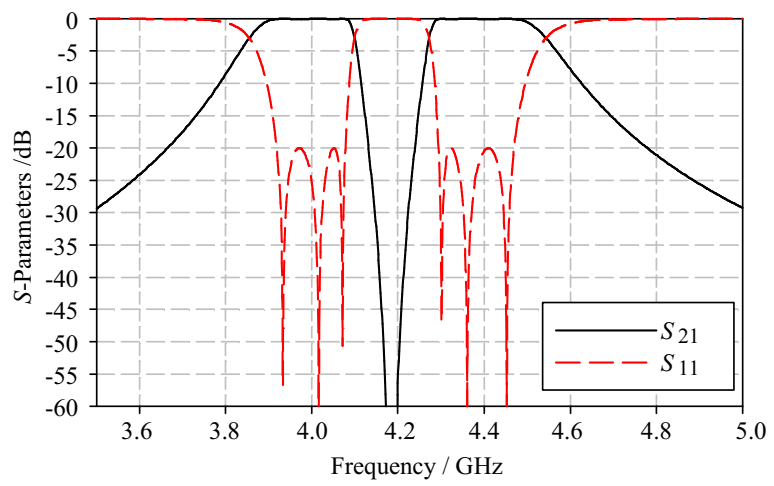

Figure 5. Simulation responses of the dual-band bandpass filter circuit model with circuit parameters as given in Table 1 , reproduced courtesy of The Electromagnetics Academy 


\section{DUAL-BAND FILTER DESIGN}

Using the procedure outlined in Section 2, the dual-band bandpass filter circuit parameters are computed and presented in Table 1 based on the specification given in Section 1. The circuit model is simulated using Agilent Advanced Design System (ADS) and the simulated results are shown in Figure 5. The simulation results show that the dual-band bandpass filter has a minimum return loss of $20 \mathrm{~dB}$ as designed and a transmission zero between the two passbands. A dual-band bandpass filter circuit model in now established.

\section{MICROSTRIP DESIGN}

To illustrate the practicality of the model developed in Section 2, a novel microstrip dual-band bandpass filter has been designed using square patch resonators. A square patch resonator will exhibit two resonant modes which are orthogonal to each other as shown in Figure 6(a). These two modes are degenerated modes, i.e., resonating

Table 1. Circuit parameters of a 6 pole dual-band bandpass filter with return loss of $20 \mathrm{~dB}$ and $50 \Omega$ terminations. (Note: the first 4 columns are the circuit parameters of a 6 pole Chebyshev filter), reproduced courtesy of The Electromagnetics Academy

\begin{tabular}{|c|c|c|c|c|}
\hline$L / \mathrm{nH}$ & $C / \mathrm{pF}$ & $J_{01}=J_{34}$ & $J_{12}$ & $J_{11^{\prime}}$ \\
\hline 0.1786 & 8.0965 & 0.02 & 0.01757 & 0.017038 \\
\hline
\end{tabular}

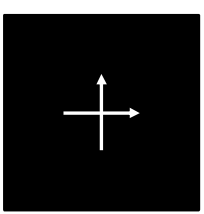

(a)

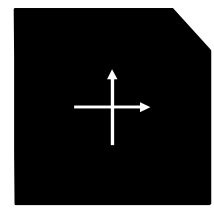

(b)

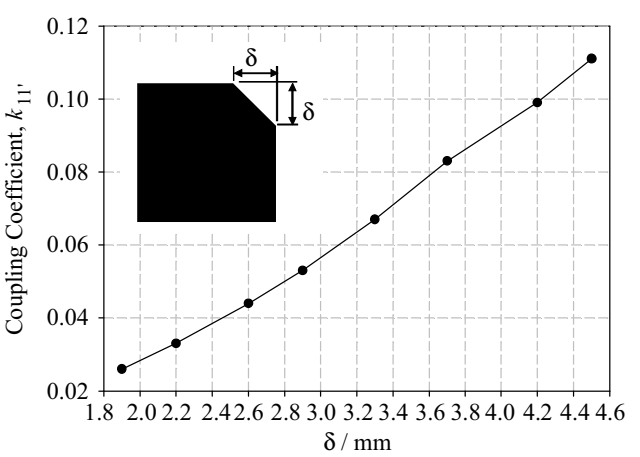

Figure 6. Square patch resonator. (a) Uncoupled orthogonal modes. (b) Coupled orthogonal modes, reproduced courtesy of The Electrogmagnetics Academy

Figure 7. Coupling coefficient of the dual-mode square patch resonator, reproduced courtesy of The Electromagnetics Academy 
at the same frequency. Because they are orthogonal, these two modes are not coupled to each other. However, the two degenerated orthogonal modes can be made to couple to each other by creating a small perturbation at one corner of the square patch $[6,7]$ as shown in Figure $6(\mathrm{~b})$. The coupling strength or the coupling coefficient between these two modes can be controlled by the amount of perturbation introduced.

To achieve the given specification, i.e., the center frequency, $f_{o}$, the square patch is designed to have a dimension of $10.97 \times 10.97 \mathrm{~mm}^{2}$. The amount of perturbation required to achieve the desired coupling coefficient between the two degenerated modes of the dual-mode square patch resonator is determined using Agilent ADS Momentum.

In the dual-band bandpass filter design, each dual-mode of a square patch resonator is a coupled resonators between resonators $x$ and $x^{\prime}$ (where $x$ is 1,2 or 3 with reference to Figure $4(\mathrm{~b})$ ). Therefore, three dual-mode resonators are sufficient in achieving the circuit model presented in Figure 4. Figure 7 shows the simulated coupling coefficients of the degenerated modes of a square patch resonator. When the perturbation increases, i.e., $\delta$ increases, the coupling strength increases. Based on (3), the coupling coefficient required to achieve the desired specification is 0.0799. From the simulated coupling graph in Figure 7 , this can be achieved with a perturbation, $\delta$, of approximately $3.6 \mathrm{~mm}$.

The next step is to determine the coupling between resonators 1 and 2. However, this cannot be easily done in a dual-mode resonator. By simulating a coupled of perfect square resonators, it will most likely overestimate the coupling strength since the final filter required perturbed square patch resonators to couple the degenerated modes rather than a perfect square patch resonators. Here, we propose a novel method to determine the coupling between resonators 1 and 2 by simulating two dual-mode square patch resonators as shown in Figure 8(a) and deduced the coupling coefficient from the eigenmodes. There are 6 coupling paths in the coupled dual-mode square patch resonators as shown in the left node diagram of Figure 8(b). However, half of the coupling paths, i.e., $k_{1^{\prime} 2}, k_{12^{\prime}}$ and $k_{1^{\prime} 2^{\prime}}$, are very much weaker compared to the other half of the coupling paths, i.e., $k_{12}, k_{11^{\prime}}$ and $k_{22^{\prime}}$, and therefore can be ignored as shown in right node diagram of Figure 8(b). With this simplification, the circuit model of the coupled dual-mode resonators can be shown as in Figure 9. To determine the eigenmodes of this circuit, the resonators must be very weakly coupled to the input and output ports to ensure that the input and output ports have minimum effect on the circuit natural resonant modes. In the circuit model, the input and output couplings, which are modeled 


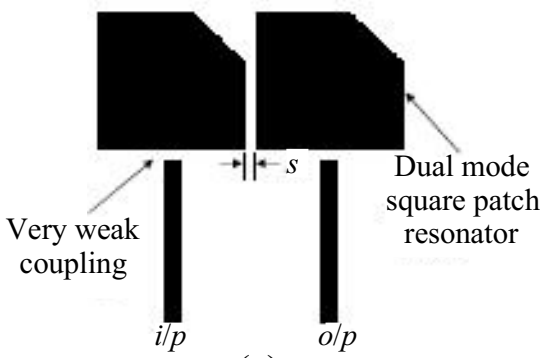

(a)

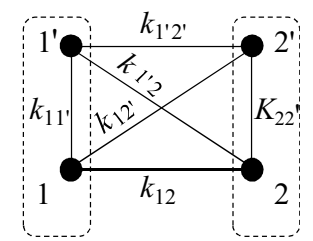

Dual-mode resonator
Dual-mode resonator

$$
\left.\begin{array}{l}
k_{12^{\prime}} \\
k_{1^{\prime} 2} \\
k_{1^{\prime} 2^{\prime}}
\end{array}\right\} \ll<k_{12} \& k_{11^{\prime}}
$$

(b)

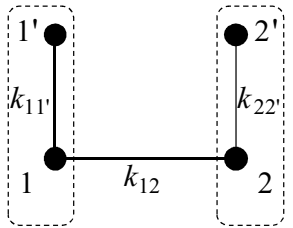

Dual-mode Dual-mode resonator resonator

Figure 8. (a) Coupled of 2 dual-mode square patch resonators. (b) The node diagram of the coupled dual-mode resonators with the associated coupling paths, reproduced courtesy of The Electromagnetics Academy

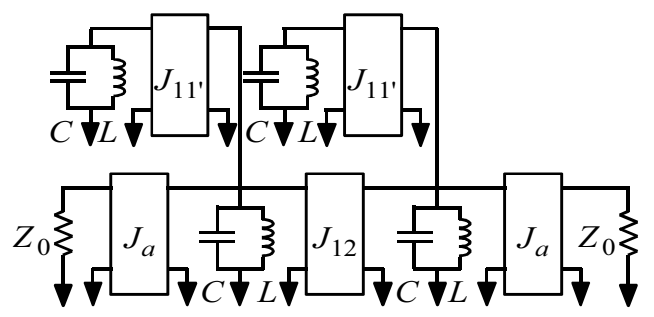

Figure 9. Circuit model for determining the eigenmodes of the dualband coupling scheme, reproduced courtesy of The Electromagnetics Academy

by $J$-inverters, $J_{a}$, with a value of 0.0001 , are 200 time weaker than the filter loading (or 200 time larger than the filter external quality factor, $Q_{\text {ext }}$ ). The relationship between the J-inverter and the $Q_{e x t}$ is given in $(4)[5,12]$. Figure 10 shows the simulated response of the circuit model in Figure 9. The coupling coefficient between resonators 1 and 2 , i.e., $k_{12}$, can be determined by (5)

$$
Q_{e x t}=\frac{\omega_{o} C}{J}
$$




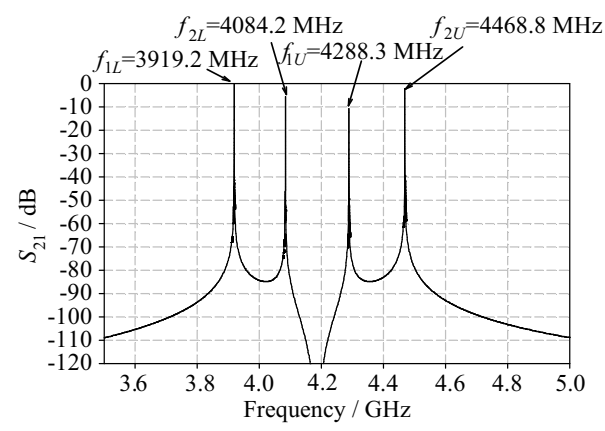

Figure 10. Simulated response of the Figure 9 with its eigenmodes labeled ${ }_{i}$ reproduced courtesy of The Electromagnetics Academy

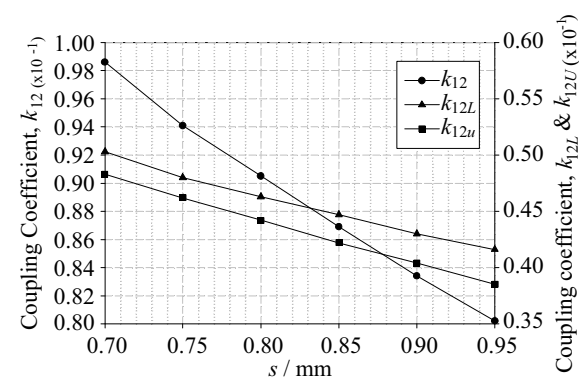

Figure 11. Simulated coupling coefficients of $k_{12}$, reproduced courtesy of The Electrogmagnetics Academy

where $J$ is the $J$-inverter value between the feed-line and the resonator.

$$
k_{12}=k_{12 L}+k_{12 U}
$$

where $k_{12 L}=\frac{f_{2 L}^{2}-f_{1 L}^{2}}{f_{2 L}^{2}+f_{1 L}^{2}} \& k_{12 U}=\frac{f_{2 U}^{2}-f_{1 U}^{2}}{f_{2 U}^{2}+f_{1 U}^{2}}$.

$f_{1 L}, f_{2 L}, f_{1 U}$, and $f_{2 U}$ are the eigenmodes, which are shown in Figure 10. From the circuit model, the coupling coefficient, $k_{12}$, can be determined by (6) [13].

$$
k_{12}=J_{12} \sqrt{\frac{L}{C}}
$$

Referring to Figure 9 , by reducing the $J_{11^{\prime}}$ parameters, the eigenmodes will alter but the coupling coefficient $k_{12}$ will not change, i.e., the sum of $k_{12 L}$ and $k_{12 U}$ will remain the same. If $J_{11^{\prime}}$ is reduced to zero in the circuit model, the model will reduce to a single mode coupled resonators which will have the same coupling coefficient as $k_{12}$. The calculated value of $k_{12}$ from the circuit model is 0.08254 using (6) whereas the $k_{12}$ extracted using (5) from the simulated eigenmodes is 0.08242 . The result shows an error of smaller than $0.15 \%$ between the two. This error can be attributed to floating point truncation of the parameters used in the simulation and also the small loading effect of the input and output ports. With such a small error, it can be safely concluded that the proposed method is valid and can be used to determine the coupling coefficient between resonators 1 and 2 from two dual-mode square patch resonators.

Having established the validity of (5), the coupling coefficient, $k_{12}$, can be extracted by simulating the structure shown in Figure 8(a). By varying the spacing, $s$, between the two dual-mode resonators while maintaining the perturbation, i.e., $\delta=3.6 \mathrm{~mm}$, the coupling coefficient, 
$k_{12}$, can be varied. A plot of the coupling coefficient, $k_{12}$, can be plotted as shown in Figure 11. The required $k_{12}$ for the dual-band filter is approximately 0.083 and from the graph, the spacing between the dual-mode resonators is determined as approximately $0.91 \mathrm{~mm}$.

For the filter loading, there are two methods can be used. There are namely, the gap coupled and the tap coupled. According to (4), the required external quality factor, $Q_{e x t}$, is 10.6 for this filter, which is relatively low, i.e., strong input/output coupling is required. Therefore, using the gap coupled method will result in extremely narrow gap which is very costly to realize in practice. Therefore, the tap coupled is used in this design instead.

The tap coupled can be achieved by directly connecting the input and output feed-lines to the first and last resonators, respectively. Conventional, the coupling strength can be altered by tapping at different current point along the resonator [14], i.e., tapping between the maximum current (center) and the minimum current (open end) of the resonator. In a square patch resonator, the ratio between the widths of the square patch resonator to the $50 \Omega$ input/output feed-line is small. Therefore, the $Q_{\text {ext }}$ is very difficult to control by using the off-center tapping method.

Here, we propose a center tapping method for square patch resonator. We believed this is a novel method for square patch resonator. However, similar method has been used for multi-mode resonator [15]. The mode that excited in the center tapping and the off-center tapping is two different modes. In the off-center tapping, the resonant mode that is excited is the mode that is orthogonal with the feed-line whereas in the center-tapping, the mode that is excited is the mode that is in parallel to the feed-line. The two different tap coupled

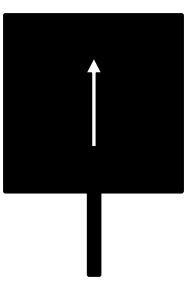

(a)

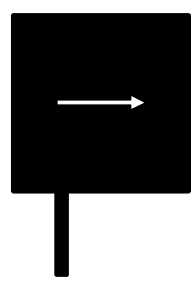

(b)

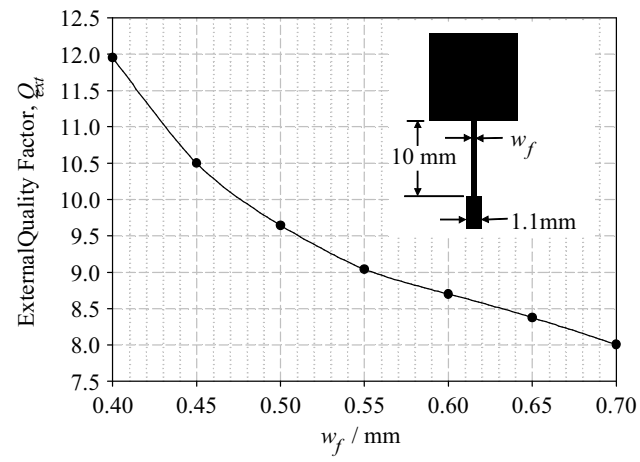

Figure 12. (a) Center tap input. (b) Off-center tap input, reproduced courtesy of The Electrogmagnetics Academy
Figure 13. External quality factor with center tap input, reproduced courtesy of The Electrogmagnetics Academy 
methods are shown in Figure 12. The white arrow shows the current direction of the resonant mode of the square patch resonator.

In the center tap coupled method, the input coupling strength is controlled by the change in the step impedance from the feed-line to the square patch line impedance. By changing the feed-line impedance, the input/output coupling can be controlled. The step impedance feed-line is then connected to a $50 \Omega$ microstrip line to ensure a $50 \Omega$ input/output termination. The length of the step impedance feed-line is very important. To ensure the step impedance feed-line does not resonate at the filter band, the length of the line has to be a multiple of half wavelength because it will resonate at a multiply of quarter wavelength.

The $Q_{\text {ext }}$ of the input to the square patch resonator can be extracted from the simulated response of a doubly loaded square patch resonator. The line-width, $w_{f}$, of the step impedance feed-line is varied to obtain a range of $Q_{\text {ext }}$. The plot of $w_{f}$ against $Q_{\text {ext }}$ is shown in Figure 13. A perfect square is used in this extraction rather than a perturbed square, which is required for the filter. This will most likely over-estimate the $Q_{\text {ext }}$ in comparison to the perturbed square. This error will be corrected by optimization process which is essential is most design procedure.

\section{EM SIMULATION RESULTS}

From the previous section, all the dimensions of the dual-band filter have been established. Figure 14 shows the layout of the microstrip dual-band bandpass filter with three dual-mode square

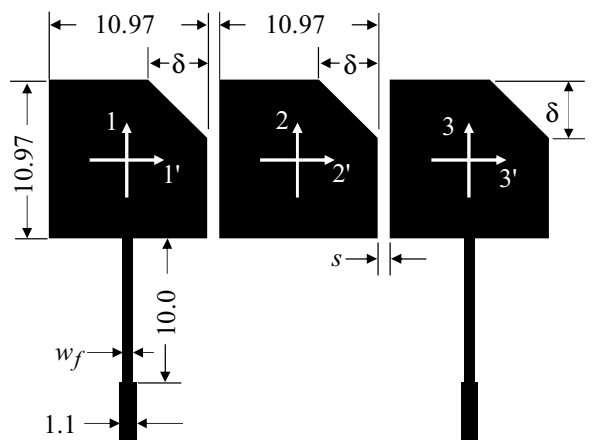

Figure 14. Dual-band bandpass filter layout (All the dimensions are in $\mathrm{mm}$ ), reproduced courtesy of The Electromagnetics Academy

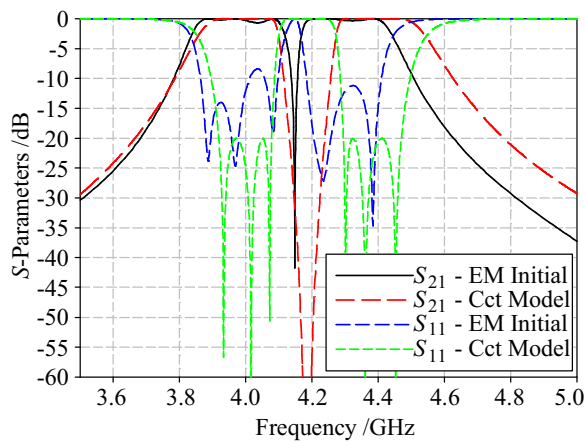

Figure 15. Comparison of the EM simulation of the initial design and the ideal circuit model, reproduced courtesy of The Electrogmagnetics Academy 
patch resonators. The microstrip filter is designed on a RT/Duroid 6010LM substrate with a substrate thickness of $1.27 \mathrm{~mm}$ and a dielectric constant of 10.8. The initial dimensions of the filter are given in Table 2. The line-width of the input and output microstrip transmission lines is $1.1 \mathrm{~mm}$ which is designed for $50 \Omega$ characteristic impedance.

The initial filter design is simulated using Agilent ADS Momentum. Figure 15 shows the EM simulation results of the initial design in comparison with the ideal circuit model. The two passbands of the initial design are closer together in comparison to the specification. The minimum return loss of the passbands is smaller than $10 \mathrm{~dB}$.

To improve the filter responses, optimization is required. Since the two passbands are closer than designed, it can be deduced that the coupling coefficient, $k_{11^{\prime}}$, in the microstrip filter circuit is weaker than the required. This can be rectified by increasing the perturbation, $\delta$ to $4.07 \mathrm{~mm}$. To improve the minimum return loss at the passbands, i.e., to reduce the passband ripples, the input and output step impedance feedlines and the spacing between the dual-mode square patch resonators are also altered. The optimized microstrip dual-band bandpass filter parameters are also shown in Table 2.

From the filter layout diagram shown in Figure 14, it is obvious

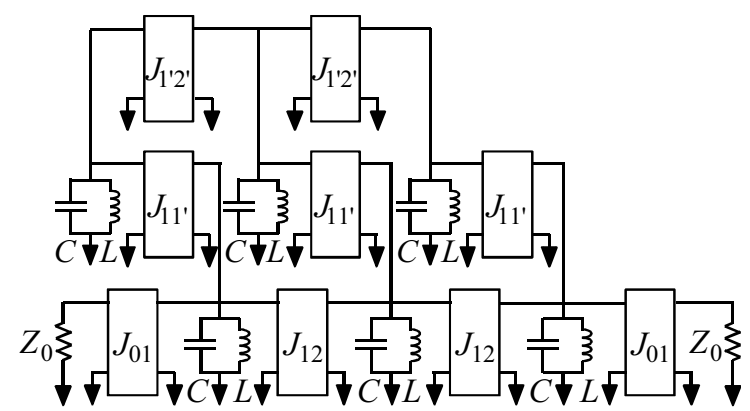

Figure 16. Circuit model of the dual-band filter with unwanted crosscoupling, which is modeled by $J_{1^{\prime} 2^{\prime}}$, reproduced courtesy of The Electromagnetics Academy

Table 2. Dimensions of the microstrip filter layout (all dimensions in $\mathrm{mm}$ ), reproduced courtesy of The Electromagnetics Academy

\begin{tabular}{|c|c|c|c|}
\hline Design & $\delta$ & $s$ & $w_{f}$ \\
\hline Initial & 3.6 & 0.91 & 0.44 \\
\hline Optimized & 4.07 & 0.85 & 0.7 \\
\hline
\end{tabular}




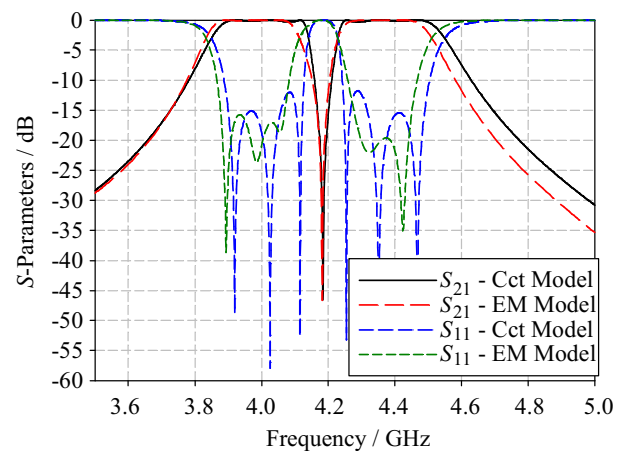

Figure 17. Comparison between the modified circuit model and the EM simulation results of the optimized design, reproduced courtesy of The Electrogmagnetics Academy

that there are unwanted couplings which are not modeled in the ideal dual-band bandpass filter circuit model. The unwanted couplings are the coupling between resonators $1^{\prime}$ and $2^{\prime}$, and resonators $2^{\prime}$ and $3^{\prime}$. To model these unwanted couplings, the circuit model is modified by introducing two additional identical $J$-inverters, $J_{1^{\prime} 2^{\prime}}$ as shown in Figure 16. The unwanted coupling coefficient, $k_{1^{\prime} 2^{\prime}}$, is quantified by EM simulation and is found to have a value of 0.01308 . This coupling coefficient is then modeled with $J_{1^{\prime} 2^{\prime}}$ with a value of 0.002785 .

Figure 17 shows the simulation results of the modified circuit model in comparison with the optimized microstrip dual-band bandpass filter. The circuit model and EM model simulations are both in ideal conditions, i.e., lossless. The results show good agreement between the circuit model and the EM simulation responses. The two passbands of the optimized design are very close to the specification. The minimum return loss of the passbands is well below $15 \mathrm{~dB}$.

\section{CIRCUIT FABRICATION}

The microstrip dual-band bandpass filter is fabricated using printed circuit board milling process. RT/Duroid 6010LM substrate which has a thickness of $1.27 \mathrm{~mm}$ and a dielectric constant of 10.8 is used for the microstrip circuit.

Figure 18 shows a photograph of the fabricated microstrip dualband bandpass filter. Two SMA connectors are fitted to the input and output of the filter for testing. The size of the filter is relatively compact, i.e., comparing it with the same order distributed resonator 


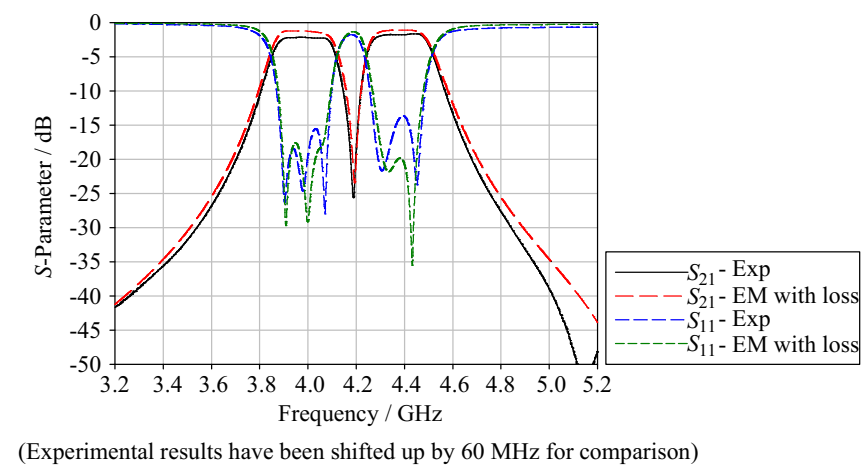

Figure 19. The EM simulated results with conductor and dielectric losses and the measured results of the microstrip dual-band dual-mode square patch resonator filter, reproduced courtesy of The Electromagnetics Academy

filter. The area size of filter in terms of the guided wavelength, $\lambda_{g}$, of the filter center frequency is measured at approximately $1.7 \lambda_{g} \times 1.2 \lambda_{g}$.

\section{EXPERIMENTAL RESULTS}

The fabricated microstrip filter is placed into an enclosed housing and is tested using Agilent Vector Network Analyzer. The measured results are presented in Figure 19 together with the EM simulated responses. The EM simulation results presented in Figure 19 is the optimized design with parameters presented in Table 2 and is simulated by taking into consideration of the conductor and dielectric losses. The measured center frequency of the filter is approximately $60 \mathrm{MHz}$ lower than the specification. This can be attributed to the tolerance of the dielectric constant of the substrate, the fabrication tolerance and also the ideal simulation condition, i.e., no surface roughness and thickness variation of the substrate are considered in the simulation. The RT/Duroid 6010LM material has a dielectric constant tolerance in the range of $\pm 2.5 \%$. A $2.5 \%$ shift upwards in the dielectric constant will result in approximately $60 \mathrm{MHz}$ downwards shift in the filter center frequency.

Other than the shift in frequency, the measured results show a very good agreement with the EM simulated results. The measured minimum passband insertion loss of the filter is approximately $1.6 \mathrm{~dB}$ and the minimum return loss at the passbands is better than $13 \mathrm{~dB}$. An additional transmission zero is also observed at around $5.09 \mathrm{GHz}$. This transmission zero is not part of the design and is generated due to unwanted cross-coupling between resonators 1 and 3 . The measured insertion loss of the passbands is slightly higher than the EM simulated 
results. The slight under-estimation of the losses in the EM simulation is most likely due to a mismatch between the actual loss parameters in the fabricated circuit and the loss parameters used in the simulation.

\section{CONCLUSION}

A novel microstrip dual-band dual-mode square patch resonator bandpass filter has been presented. A detailed design procedure starting from the normalized Chebyshev low-pass filter to a final optimized microstrip dual-band bandpass filter is also presented. The design procedure has been verified experimentally. The measured filter achieved a low insertion loss with reasonable good matching at the passbands.

The presented dual-band design circuit model is also very versatile and can be easily controlled. For example, the separation between passbands can be easily controlled, i.e., increase or decrease by simply changing the coupling, $k_{11^{\prime}}$. The bandwidth of the passbands is purely controlled by the external quality factor and the coupling, $k_{12}$, which is basically the conventional bandpass filter parameters. And therefore, the bandwidth and the separation of the passbands can be controlled independently in the presented dual-band circuit model.

\section{ACKNOWLEDGMENT}

The authors would like to Bernie Hanreck from the University of East London for his technical assistance.

\section{REFERENCES}

1. Tsai, L.-C. and C.-W. Hsue, "Dual-band bandpass filters using equal-length coupled-serial-shunted lines and $Z$-transform technique," IEEE Trans. Microwave Theory \& Tech., Vol. 52, No. 4, 1111-1117, 2004.

2. Cheng, S.-F., Y.-L. Jeng, and J.-L. Chen, "Dual-band stepimpedance bandpass filter for multimode wireless LANs," Electronics Letters, Vol. 40, No. 1, 38-39, 2004.

3. Guan, X., Z. Ma, P. Cai, Y. Kobayashi, T. Anada, and G. Hagiware, "Synthesis of dual-band bandpass filters using successive frequency transformation and circuit conversions," IEEE Microwave and Wireless Components Letters, Vol. 16, No. 3, 2006. 
4. Kuo, J.-T. and H.-S. Cheng, "Design of quasi-elliptic function filters with a dual-passband response," IEEE Microwave and Wireless Components Letters, Vol. 14, No. 10, 2004.

5. Yeo, K. S. K. and M. J. Lancaster, "8 pole high temperature superconductor microstrip dual band filter desgin," IEEE MTT-S IMS Digest, 2011.

6. Curtis, J. A. and S. J. Fiedziuszko, "Miniature dual mode microstrip filters," IEEE MTT-S IMS Digest, Vol. 2, 443-446, 1991.

7. Fiedziuszko, S. J., J. A. Curtis, S. C. Holme, and R. S. Kwok, "Low loss multiplexers with planar dual mode HTS resonators," IEEE Trans. Microwave Theory \& Tech., Vol. 44, No. 7, 12481257, 1996.

8. Cassinese, A., A. Andreone, M. Barra, C. Granata, P. Orgiani, F. Palomba, G. Panariello, G. Pica, and F. Schettino, "Dual mode superconducting planar filters based on slotted square resonators," IEEE Trans. Applied Superconductivity, Vol. 11, No. 1, 473-476, 2001.

9. Li, J.-L., J.-P. Wang, X.-S. Yang, and B.-Z. Wang, "A study of dual-mode patch resonator-based microwave filters," International Conference on Microwave and Millimeter Wave Tech. (ICMMT), 48-51, 2010.

10. Cohn, S. B., "Direct-coupled-resonator filters," Proceedings of the IRE, Vol. 45, No. 2, 187-196, 1957.

11. Matthaei, G., L. Young, and E. M. T. Jones, Microwave Filters, Impedance-matching Networks, and Coupling Structures, Artech House, Boston, 1964.

12. Yeo, K. S. K. and P. Vijaykumar, "Quasi-elliptic microstrip bandstop filter using tap coupled open-loop resonators," Progress In Electromagnetics Research C, Vol. 35, 1-11, 2013.

13. Yeo, K. S. K., M. J. Lancaster, and J.-S. Hong, "The design of microstrip six-pole quasi-elliptic filter with linear phase response using extracted-pole technique," IEEE Trans. Microwave Theory \& Tech., Vol. 49, No. 2, 321-327, 2002.

14. Yeo, K. S. K. and M. J. Lancaster, "A novel tap input coupling structure for a narrow bandpass filter using $\mathrm{TM}_{010}$ mode of a microstrip circular disk resonator," IEEE Trans. Microwave Theory \& Tech., Vol. 50, No. 4, 1240-1232, 2002.

15. Zhu, R. and L. Li, "Compact UWB bandpass filter using stubloaded multiple-mode resonator," IEEE Microwave and Wireless Components Letters, Vol. 17, No. 1, 40-42, 2007. 